Pacific Journal of Mathematics 


\title{
CONNECTEDNESS RELATED TO ALMOST PERIODICITY OF COMPOSITIONS OF FLOW HOMOMORPHISMS
}

\author{
Doug C. McMahon, JAAP Van Der Woude and Ta-Sun Wu
}

Consider homomorphisms $\phi: X \rightarrow Y$ and $\psi: Y \rightarrow Z$, where $\phi$ is open and $N$-to-one, $\psi$ is almost periodic. In the paper by $\mathbf{R}$. J Sacker and G. R. Sell, it was shown that, under a certain condition on the phase group, the composition $\psi \circ \phi: X \rightarrow Z$ is almost periodic (provided that $Z$ is trivial and $X$ is minimal). In this paper almost periodicity of $\psi \circ \phi$ is studied under connectedness conditions on the fibers of $\psi$. For instance it is shown that if $\psi$ is almost periodic with connected fibers than $\psi \cdot \phi$ is almost periodic. If $\psi$ is locally almost periodic with locally connected fibers then $\phi \circ \psi$ is locally almost periodic.

0. Introduction. The results in this paper contribute to a list of achievements with respect to the question, when is the composition of almost periodic extensions again almost periodic? In general such a composition is not almost periodic (viz: the existence of distal non-almost periodic flows). But under certain connectedness conditions on the maps involved ([E 69] page 56, [W 75]) and/or under a certain assumption for the phase group ([SS 74], [MW 76], [B 75 / 79], [R ?]) some compositions are.

The impetus for this research came from [SS 74], where it was shown an open $\mathrm{N}$-to-one extension of an almost periodic flow was an almost periodic flow again as long as the phase group was semicompactly generated (i.e., there is a compact $K \subseteq T$ such that every open $V \supseteq K$ generates $T$ ). In [MW 76] this result was strengthened to the relativized nonmetric case, while a connection was made with connectedness conditions on the fibers. This resulted in the theorem that, for a semicompactly generated phase group, minimal distal flows with 0-dimensional phase space are almost periodic. In [W 75] it was shown, without conditions on the phase group, that an open $N$-to-one extension of a connected almost periodic regular minima flow was almost periodic. Various results along this line are obtained in [B 75/79] and [R ?] with respect to manifold flows and Lie group extensions; but the conditions imposed on the phase groups and phase spaces are rather strong, mostly due to the perspective of applications. 
This paper is concerned with replacing the phase group assumption by a connectedness assumption on the fibers of the basic almost periodic extension, such that after extending by an open $N$-to-one extension we still have an almost periodic extension. Minimality is not always assumed.

In $\$ 1$ we establish some notions and notations and list some known results for the convenience of the reader. Section 2 treats the problem without minimality requirements and the main result are 2.2 (absolute case for connectedness) and 2.5 (relativized case for locally connectedness). Under the assumption of minimality we prove the relativized version of 2.2 in $\S 3$. Open $N$-to-one extension of locally almost periodic locally connected extension are studied in $\$ 4$. And finally, we give some counterexamples in $\S \S 5$ and 6 . In addition, $\S 6$ contains some results on the regional proximal relations in a suspension of a flow.

This paper results from joint efforts of the authors during the visit of the first two authors to Case Western Reserve University in the Spring Semester of 1984.

1. Preliminaries. Although we assume basic knowledge about topological dynamics, as can be found for instance in [GH 55] and [E 69], it seems useful to review some of the basic notions and establish notation.

Throughout the paper we fix a topological group $T$ and we consider (left) actions of $T$ on compact $T_{2}$ topological spaces ( $X$ with unique uniformity $\left.\mathscr{U}_{X}\right)$. A homomorphism $\phi:\langle T, X\rangle \rightarrow\langle T, Y\rangle$, or just $\phi: X \rightarrow$ $Y$, is a continuous surjection that commutes with the actions of $T$ on $X$ and $Y$. Such a homomorphism $\phi$ is defined by and defines a closed $T$-invariant equivalence relation $R_{\phi}$ on $X, R_{\phi}:=\left\{\left(x_{1}, x_{2}\right) \mid \phi\left(x_{1}\right)=\right.$ $\left.\phi\left(x_{2}\right)\right\}$. Let $R_{\phi} \cap \mathscr{U}_{X}$ denote the relative uniformity. Given a homomorphism $\phi: X \rightarrow Y$ the following closed invariant equivalence relations are considered:

$$
P_{\phi}:=\bigcap\left\{\alpha \cap R_{\phi} \mid \alpha \in \mathscr{U}_{X}\right\} \text {, or }\left(x_{1}, x_{2}\right) \in P_{\phi} \text { iff }\left(x_{1}, x_{2}\right) \in R_{\phi} \text { and }
$$
there are $t_{\lambda} \in T$ with $t_{\lambda}\left(x_{1}, x_{2}\right) \rightarrow(\bar{x}, \bar{x})$ for a certain $\bar{x} \in X$; the proximal relation for $\phi$.

$$
Q_{\phi}:=\bigcap\left\{\overline{T \alpha \cap R_{\phi}} \mid \alpha \in \mathscr{U}_{X}\right\} \text {, or }\left(x_{1}, x_{2}\right) \in Q_{\phi} \text { iff }\left(x_{1}, x_{2}\right) \in R_{\phi} \text { and }
$$
there are $t_{\lambda} \in T,\left(x_{1}^{\lambda}, x_{2}^{\lambda}\right) \in R_{\phi}$ with $\left(x_{1}^{\lambda}, x_{2}^{\lambda}\right) \rightarrow\left(x_{1}, x_{2}\right)$ and $t_{\lambda}\left(x_{1}^{\lambda}, x_{2}^{\lambda}\right)$ $\rightarrow(\bar{x}, \bar{x})$ for some $\bar{x} \in X$; the regionally proximal relation for $\phi$.

$E_{\phi}$ : the smallest closed invariant equivalence relation in $R_{\phi}$ that contains $Q_{\phi}$; the equicontinuous structure relation.

Then $\phi$ is distal (proximal) iff $P_{\phi}=\Delta_{X}\left(P_{\phi}=R_{\phi}\right)$; and $\phi$ is almost periodic iff $Q_{\phi}=\Delta_{X}$ or equivalently iff for every $\alpha \in \mathscr{U}_{X}$ there is a $\beta \in \mathscr{U}_{X}$ with $T\left(\beta \cap R_{\phi}\right) \subseteq \alpha$. If $Y$ is trivial we denote $P_{X}, Q_{X}$ and $E_{X}$. Note that in case $Y$ is trivial (the absolute case), almost periodicity for $\phi$ 
(or just $X$ ) is equivalent to the existence of a syndetic subset $B_{\alpha} \subseteq T$ for $\alpha \in \mathscr{U}_{X}$ such that $B_{\alpha} X \subseteq \alpha(x)$ for every $x \in X$.

Another important closed invariant equivalence relation for $\phi$ is

$$
\begin{aligned}
& R c(\phi)=\left\{\left(x_{1}, x_{2}\right) \in R_{\phi} \mid x_{1} \text { and } x_{2}\right. \text { are in the same connected } \\
& \text { component of the fiber } \left.\phi^{-} \phi\left(x_{1}\right)\right\} .
\end{aligned}
$$

Then $R c(\phi)$ defines maps $\kappa: X \rightarrow X / R c(\phi)$ and $\theta: X / R c(\phi) \rightarrow Y$ such that $\phi=\theta \circ \kappa$, where $\kappa$ has connected fibers and $\theta$ has totally disconnected fibers.

There exists a universal point transitive flow $S_{T}$ for $T$ that acts as a semigroup on every flow $X$ for $T$ such that $\overline{T x}=S_{T} x$. The minimal left ideals in $S_{T}$ are all isomorphic and they are isomorphic to the universal minimal flow $M$ for $T$, and $M$ acts as a semigroup on $X$ such that every minimal orbit closure $\overline{T x}$ in $X$ is just $M x$. A point with a minimal orbit closure is called an almost periodic point. Let $J$ be the collection of idempotents in $M$. Then $J \neq \varnothing$ and $x \in X$ is an almost periodic point iff $x=u x$ for some $u \in J$.

If $X$ is a flow for $T$, then $X$ induces a flow $\left\langle T, 2^{X}\right\rangle$ where $2^{X}$ is the collection of nonempty closed subset of $X$, endowed with the Vietoris topology. A subbase for the open sets in the Vietoris topology is formed by the sets of the form $\langle U\rangle$ and $\langle U\rangle^{*}$, where $U \subseteq X$ is open and

$$
\langle U\rangle:=\left\{A \in 2^{X} \mid A \subseteq U\right\} ; \quad\langle U\rangle^{*}=\left\{A \in 2^{X} \mid A \cap U \neq \varnothing\right\} .
$$

Denote the action of $S_{T}$ on $2^{X}$ by "。", the circle operation. For the convenience of the reader, we now state some results that are known or easy to prove.

1.1. Note: If $\phi: X \rightarrow Y$ is an open $N$-to-one extension, then $\phi$ is almost periodic.

1.2. LEMMA. Let $\phi: X \rightarrow Y$ and $\psi: Y \rightarrow Z$ be homomorphisms of flows. If $R_{\phi}$ is clopen in $R_{\psi \circ \phi}$ then $Q_{\phi}=Q_{\psi \circ \phi}$. In particular, if $R_{\phi}$ is clopen in $R_{\psi \circ \phi}$ and $\phi$ is almost periodic then $\psi \circ \phi$ is almost periodic.

Proof. Since $R_{\phi}$ is clopen in $R_{\phi \circ \phi}$, there is an $\alpha_{0} \in \mathscr{U}_{X}$ with $\overline{T \alpha \cap R_{\psi \circ \phi}} \subseteq R_{\phi}$ and so $\overline{T \alpha \cap R_{\psi \circ \phi}}=\overline{T \alpha \cap R_{\phi}}$ for every $\alpha \subseteq \alpha_{0}$. Hence $Q_{\phi}=Q_{\psi \circ \phi}$.

The next Theorem (1.3) is a non-minimal version of [MW 76] 2.1. (compare [Wo 82] I 1.22). 
1.3. TheOREM. If $\phi: X \rightarrow Y$ is an almost periodic extension and $\psi$ : $Y \rightarrow Z$ is open and $N$-to-one, then $\psi \circ \phi$ is almost periodic.

The next theorem is just a restatement of [MW 76] 2.6. in the nonminimal case. Remember that $\phi: X \rightarrow Y$ is a quotient group extension if there is a bitransformation group $\langle T, W, K\rangle$ with $K$ a compact $T_{2}$ topological group acting freely on $W$ such that $\langle T, Y\rangle \simeq\langle T, W / K\rangle$, and there exists a closed subgroup $L$ of $K$ such that $\langle T, X\rangle \simeq\langle T, W / L\rangle$ and $\phi:\langle T, X\rangle \rightarrow\langle t, Y\rangle$ may be identified with the canonical quotient map $\langle T, W / L\rangle \rightarrow\langle T, W / K\rangle$.

$$
\begin{array}{ccc}
\langle T, W, K\rangle & \rightarrow & \langle T, W / K\rangle \simeq\langle T, Y\rangle \\
\downarrow & \simeq & \nearrow \phi \\
\langle T, W / L\rangle & \simeq & \langle T, X\rangle
\end{array}
$$

1.4. TheOREM. If $\phi: X \rightarrow Y$ is a quotient group extension with totally disconnected fibers, then $\phi$ is the inverse limit of open finite-to-one extensions $\phi_{N}: X_{N} \rightarrow Y$ (where $N$ is the size of the fiber).

We end this section with a lemma that is basic in all results to follow, namely 3.3 from [MW 76]. We provide the proof for the convenience of the reader since the proof in [MW 76] had an unfortunate although not severe gap. The Lemma is purely topological.

1.5. LemMa. Let $\phi: X \rightarrow Y$ be an open $N$-to-one continuous map. Then there exists an open $\alpha \in \mathscr{U}_{X}$ such that for every $\gamma \in \mathscr{U}_{X}$ with $\gamma \subseteq \alpha$ there is a $\beta \in \mathscr{U}_{Y}$ such that

$$
(\phi \times \phi)^{\leftarrow}(\beta) \subseteq \gamma \cup \alpha^{c}
$$

Proof. For each $y \in Y$ denote $\phi^{\leftarrow}(y)=\left\{x_{y}^{i} \mid i=1, \ldots, N\right\}$. Since $\phi$ is open and $N$-to-one, for every $y \in Y$ we can find open neighbourhoods $V_{y}$ of $y$ in $Y$ and $W_{y}^{i}$ of $x_{y}^{i}$ in $X$ such that $\phi^{\leftarrow}\left[V_{y}\right]=\bigcup\left\{W_{y}^{i} \mid i=1, \ldots, N\right\}$ where $x_{y}^{i} \in W_{y}^{i}$ and $\left.\phi\right|_{W_{y}^{i}}: W_{y}^{i} \rightarrow V_{y}$ is a homeomorphism.

Let $\alpha_{y} \in \mathscr{U}_{X}$ be such that $\alpha_{y}^{3}\left(x_{y}^{i}\right) \subseteq W_{y}^{i}$ and $\alpha_{y}=\alpha_{y}^{-1}$. Then $U_{y}:=\bigcap\left\{\phi\left[\alpha_{y}\left(x_{y}^{i}\right)\right] \mid i=1, \ldots, N\right\}$ is an open neighbourhood of $y$ in $Y$. Choose $y_{1}, \ldots, y_{n}$ in $Y$ such that $Y=\bigcup\left\{U_{y_{j}} \mid j=1, \ldots, n\right\}$ and let $\alpha$ : $\subseteq \bigcap\left\{\alpha_{y_{j}} \mid j=1, \ldots, n\right\}$ : with $\alpha=\alpha^{-1}$. Since $\Delta_{y} \subseteq \bigcup\left\{U_{y_{j}} \times U_{y_{j}} \mid j=\right.$ $1, \ldots, n\}$ there is a $\delta \in \mathscr{U}_{y}$ such that $\left(y, y^{\prime}\right) \in \delta$ implies $\left(y, y^{\prime}\right) \in U_{y_{J}} \times U_{y_{J}}$ for some $j \in\{1, \ldots, n\}$.

Choose $\gamma \in \mathscr{U}_{X}$ with $\gamma \subseteq \alpha$ and define $\nu:=\left\{\left(y, y^{\prime}\right) \in Y \times Y \mid\{x\} \times \phi^{\leftarrow}\left(y^{\prime}\right) \cap \gamma \neq \varnothing\right.$ for every $\left.x \in \phi^{\leftarrow}(y)\right\}$. 
Since $\phi$ is open, $\phi_{a d}$ is continuous, so the map

$$
\left(x, x^{\prime}\right) \mapsto\{x\} \times \phi^{\leftarrow} \phi\left(x^{\prime}\right): R_{\phi} \rightarrow 2^{R_{\phi}}
$$

is (lower semi) continuous, so again by openness of $\phi$ it follows that $\nu \in \mathscr{U}_{Y}$ (hint: look at $\left\langle\gamma \cap R_{\phi}\right\rangle^{*}$, subbase open in $2^{R_{\phi}}$ ). Define $\beta:=\delta \cap$ $\nu \in \mathscr{U}_{Y}$.

Let $\left(\phi(x), \phi\left(x^{\prime}\right)\right) \in \beta$, then $\left(\phi(x), \phi\left(x^{\prime}\right)\right) \in U_{y_{j}} \times U_{y_{j}}$ for some $j \in$ $\{1, \ldots, n\}$; so for certain $k, L \in\{1, \ldots, N\}$ we have $\left(x, x^{\prime}\right) \in \alpha_{y_{j}}\left(x_{y_{j}}^{k}\right) \times$ $\alpha_{y_{j}}\left(x_{y_{j}}^{L}\right)$.

If $k \neq L$ then $\left(x, x^{\prime}\right) \notin \alpha$. For: suppose $\left(x, x^{\prime}\right) \in \alpha$ then

$$
x_{y_{j}}^{k} \in \alpha_{y_{j}} \circ \alpha \circ \alpha_{y_{j}}\left(x_{y_{j}}^{L}\right) \subseteq \alpha_{y_{j}}^{3}\left(x^{L}\right) \subseteq W_{y_{j}}^{L} .
$$

So $W_{y_{j}}^{k} \cap W_{y_{j}}^{L} \neq \varnothing$, contradicting the choice of $V_{y_{j}}$. If $k=L$, then $\left(x, x^{\prime}\right) \in W_{y_{j}}^{k_{J}} \times W_{y_{j}}^{k}$. Since $\left(\phi(x), \phi\left(x^{\prime}\right)\right) \in \nu$ there is an $x^{\prime \prime} \in \phi^{\leftarrow} \phi\left(x^{\prime}\right)$ with $\left(x, x^{\prime \prime}\right) \in \gamma$, so

$$
x^{\prime \prime} \in \gamma(x) \subseteq \alpha(x) \subseteq \alpha_{y_{j}}^{3}\left(x_{y_{j}}^{k}\right) \subseteq W_{y_{j}}^{k} .
$$

since $W_{y_{\jmath}}^{k} \stackrel{\phi}{\rightarrow} V_{y_{j}}$ is one to one it follows that $x^{\prime}=x^{\prime \prime}$ and so that $\left(x, x^{\prime}\right)=\left(x, x^{\prime \prime}\right) \in \gamma$.

2. The nonminimal case. In this section we discuss two situations in which an open $\mathrm{N}$-to-one extension of an almost periodic extension is almost periodic. The results do not require minimality for the flows involved (except for $Z$ in Theorem 2.5). In the minimal case we can give a relativized version of 2.2 , which immediately covers the minimal case for 2.5. This will be done in $\S 3$.

First we need a lemma; the proof depends on [Sh 76] 1.4. Note that in the proof of [Sh 76] 1.4 only pointwise almost periodicity of the factor space $Y$ is required (iii $\Rightarrow$ ii).

2.1. LemMa. Let $\phi: X \rightarrow Y$ be an almost periodic extension and let $Y$ be pointwise almost periodic. Then for $y=u y \in Y$ and for every $\alpha \in \mathscr{U}_{X}$ there is an open neighbourhood $U$ of $u$ in $M$ such that $U x \subseteq \alpha(x)$ for every $x \in \phi^{\leftarrow}(y)$.

Proof. Choose $\gamma, \delta \in \mathscr{U}_{X}$ with $\delta \subseteq \bar{\delta} \subseteq \gamma=\gamma^{-1} \subseteq \gamma^{2} \subseteq \alpha$. Choose finitely many $x_{i} \in \phi^{\leftarrow}(y)$ such that $\phi^{\leftarrow}(y) \subseteq \cup \delta\left(x_{i}\right)$. Since $\phi$ is almost periodic, the closed set $\bar{\delta}\left(x_{i}\right) \cap \phi^{\leftarrow}(y)$ is an almost periodic point in $2^{X}$ ([Sh 74] 1.4). Since $\bar{\delta}\left(x_{i}\right) \cap \phi^{\leftarrow}(y) \subseteq \gamma\left(x_{i}\right), \bar{\delta}\left(x_{i}\right) \cap \phi^{\leftarrow}(y) \in\left\langle\gamma\left(x_{i}\right)\right\rangle$, there is an open neighbourhood $U_{i}$ of $u$ such that

$$
U_{i} \circ\left(\bar{\delta}\left(x_{i}\right) \cap \phi^{\leftarrow}(y)\right)=\left\{\alpha \circ\left(\bar{\delta}\left(x_{i}\right) \cap \phi^{\leftarrow}(y)\right) \mid \alpha \in U_{i}\right\} \subseteq\left\langle\gamma\left(x_{i}\right)\right\rangle .
$$

Hence $U_{i} \cdot\left(\bar{\delta}\left(x_{i}\right) \cap \phi^{\leftarrow}(y)\right) \subseteq \gamma\left(x_{i}\right)$. 
Define $U=\cap U_{i}$, then $U$ is an open neighbourhood of $u$ in $M$. Let $x \in \phi^{\leftarrow}(y)$. Then for some $i, x \in \delta\left(x_{i}\right) \cap \phi^{\leftarrow}(y)$. So

$$
U x \subseteq U_{i} x \subseteq U_{i} \circ\left(\bar{\delta}\left(x_{i}\right) \cap \phi^{\leftarrow}(y)\right) \subseteq \gamma\left(x_{i}\right) .
$$

Since $x \in \delta\left(x_{i}\right) \subseteq \gamma\left(x_{i}\right)$ and $\gamma=\gamma^{-1}$ we have $x_{i} \in \gamma(x)$. Hence

$$
U x \subseteq \gamma\left(x_{i}\right) \subseteq \gamma^{2}(x) \subseteq \alpha(x) .
$$

In the proof of the next theorem we use the same basic technique used in 1.5 of [ W 75].

2.2. THEOREM. Let $Y$ be an almost periodic flow with a finite number of (connected) components and let $\phi: X \rightarrow Y$ be an open $N$-to-one extension. Then $X$ is an almost periodic flow.

Proof. First note that $X$ is distal and pointwise almost periodic and that $X$ has a finite number of components, say $X=\bigcup\left\{C\left(x_{i}\right) \mid i=\right.$ $1, \ldots, m\}$ where $C\left(x_{i}\right)$ is the component of $x_{i}$ in $X$.

Let $\gamma \in \mathscr{U}_{X}$ be open. We show that there is a syndetic subset $B \subseteq T$ such that $B x \subseteq \gamma(x)$ for every $x \in X$, which in turn shows the almost periodicity of $X$.

Let $\alpha \in \mathscr{U}_{X}$ be as in Lemma 1.5, and without loss of generality let $\bar{\gamma} \subseteq \alpha$. By Lemma 1.5 we can find $\beta \in \mathscr{U}_{Y}$ such that $(\phi \times \phi)^{\leftarrow}(\beta) \subseteq \gamma$ $\cup \alpha^{c}$. Let $u \in J$. Since $Y$ is almost periodic, $Y=u Y$, there is an open neighbourhood $U$ of $u$ in $M$ such that $U y \subseteq \beta(y)$ for every $y \in Y$ by 2.1. Since $X$ is distal and pointwise almost periodic, $\left(x_{1}, \ldots, x_{m}\right)$ is an almost periodic point in $X^{m}$. So there is an open neighbourhood $U^{\prime}$ of $u$ in $M$ such that

$$
U^{\prime}\left(x_{1}, \ldots, x_{m}\right) \subseteq \gamma\left(x_{1}\right) \times \cdots \times \gamma\left(x_{m}\right) .
$$

Let $B=\left\{t \in T \mid t u \in U \cap U^{\prime}\right\}$, then $B$ is syndetic in $T$ and $B y \subseteq \beta(y)$ for every $y \in Y$ while $B x_{i} \subseteq \gamma\left(x_{i}\right)$ for every $x_{i} \in X, i \in\{1, \ldots, m\}$. Fix $i \in\{1, \ldots, m\}$ and set $C=C\left(x_{i}\right)$. Define for every $b \in B$ the set

$$
A(b):=\{x \in C \mid(x, b x) \in \gamma\} .
$$

By the construction of $B$ we have $x_{i} \in A(b)$ so $A(b) \neq \varnothing$. Since $\gamma$ is open, $A(b)$ is open. Also, $A(b)$ is closed, as follows: Let $(x, b x) \in \bar{\gamma} \subseteq \alpha$. Since $(\phi(x), \phi(b x))=(\phi(x), b \phi(x)) \in \beta$ (construction of $B)$, it follows that $(x, b x) \in \gamma($ Lemma 1.5). So $A(b)=\{x \in C \mid(x, b x) \in \bar{\gamma}\}$ and $A(b)$ is closed. By the connectedness of $C$, we have $C=A(b)$, i.e., $b x \in \gamma(x)$ for every $x \in C$. Since this holds for every $b \in B$ and every $C\left(x_{i}\right)$, it follows that $B x \subseteq \gamma(x)$ for every $x \in X$. 
2.3. Corollary. Let $\phi: X \rightarrow Y$ be a quotient group extension with totally disconnected fibers, let $\psi: Y \rightarrow Z$ be almost periodic with connected fibers and let $Z$ be finite. Then $X$ is an almost periodic flow.

Proof. Since $Z$ is finite, it follows from 1.3 that $Y$ is almost periodic and since $\psi$ has connected fibers, $Y$ has a finite number of components. By 1.4, $\phi$ is the inverse limit of finite-to-one extensions $\phi_{N}: X_{N} \rightarrow Y$. From 2.2 we know that every $X_{N}$ is almost periodic and so $X=\operatorname{inv} \lim X_{N}$ is almost periodic.

Note that Theorem 2.2 is not valid in the nonminimal relativized case as is shwon in example $\$ 5$. If connectedness is replaced by locally connectedness, however, relativization is possible.

Before we come to the theorem we need the following lemma.

2.4. LEMMA. Let $\psi: Y \rightarrow Z$ be an almost periodic extension and let $Z$ be minimal, $z \in Z$. Let $V_{1}, \ldots, V_{k}$ be nonempty open sets in $\psi^{\leftarrow}(z)$ that cover $\psi^{\leftarrow}(z)$. Then

$$
W:=\bigcup_{i=1}^{k} \overline{T\left(V_{i} \times V_{i}\right)} \in R_{\psi} \cap \mathscr{U}_{Y} .
$$

If $Y$ is minimal and $V$ is an open set in $\psi^{\leftarrow}(z)$, then

$$
W:=\overline{T(V \times V)} \in R_{\psi} \cap \mathscr{U}_{Y} .
$$

Proof. Since $Z$ is minimal and $\psi$ is open it is easily shown that $R_{\psi}$ $=\overline{T\left(\psi^{\leftarrow}(z) \times \psi^{\leftarrow}(z)\right)}$. Choose open sets $U_{i}$ in $Y$ such that $V_{i}=U_{i} \cap$ $\psi^{\leftarrow}(z)$, and let $U=\bigcup\left\{U_{i} \mid i=1, \ldots, k\right\}$. Let $\mu \in \mathscr{U}_{Y}$ be such that

$$
\mu \cap \psi^{\leftarrow}(z) \times \psi^{\leftarrow}(z) \subseteq \bigcup\left\{U_{i} \times U_{i} \mid i=1, \ldots, k\right\} .
$$

[For every $y \notin U$ choose an open neighbourhood $U_{y}$ of $y$ with $U_{y} \cap \psi^{\leftarrow}$ $(z)=\phi$. Then $\bigcup\left\{U_{i} \times U_{i} \mid i=1, \ldots, k\right\} \cup \bigcup\left\{U_{y} \times U_{y} \mid y \notin U\right\} \in \mathscr{U}_{Y}$.] By almost periodic there is a $\mu^{\prime} \in \mathscr{U}_{Y}$ with $T \mu^{\prime} \cap R_{\psi} \subseteq \mu$. Let $y \in Y$, we show that $(y, y) \in \operatorname{int}_{R_{\psi}} W$. Suppose $(y, y) \notin \operatorname{int}_{R_{\psi}} W$. Then there are nets $\left(y_{\lambda}^{1}, y_{\lambda}^{2}\right) \in \psi^{\leftarrow}(z) \times \psi^{\leftarrow}(z), t_{\lambda} \in T$, such that $t_{\lambda}\left(y_{\lambda}^{1}, y_{\lambda}^{2}\right) \rightarrow(y, y)$ while $t_{\lambda}\left(y_{\lambda}^{1}, y_{\lambda}^{2}\right) \notin W$, hence $\left(y_{\lambda}^{1}, y_{\lambda}^{2}\right) \notin W$. Since $t_{\lambda}\left(y_{\lambda}^{1}, y_{\lambda}^{2}\right) \rightarrow(y, y)$, $t_{\lambda}\left(y_{\lambda}^{1}, y_{\lambda}^{2}\right) \in \mu^{\prime}$ for every $\lambda \geq \lambda_{0}$. Hence $\left(y_{\lambda}^{1}, y_{\lambda}^{2}\right) \in \mu$ for $\lambda \geq \lambda_{0}$. After passing to a suitable subnet, $\left(y_{\lambda}^{1}, y_{\lambda}^{2}\right) \in U_{j} \times U_{j}$ for a certain $j \in$ $\{1, \ldots, k\}$. But as $U_{j} \cap \psi^{-}(z)=V_{j}$ it follows that $\left(y_{\lambda}^{1}, y_{\lambda}^{2}\right) \in W$ contradicting the choice of $\left(y_{\lambda}^{1}, y_{\lambda}^{2}\right)$. Consequently, $(y, y) \in$ int $_{R_{\psi}} W$; thus $W \in R_{\psi} \cap \mathscr{U}_{Y}$.

If $Y$ is minimal, $R_{\psi}=\overline{T\left(\left\{y_{0}\right\} \times \psi^{\leftarrow}(z)\right)}$ for every $y_{0} \in \psi^{\leftarrow}(z)$. So let $y_{0} \in V$, let $\mu \in \mathscr{U}_{Y}$ be such that $\left\{y_{0}\right\} \times \mu\left(y_{0}\right) \cap R_{\psi} \subseteq\left\{y_{0}\right\} \times V$. Let $\mu^{\prime} \in \mathscr{U}_{Y}$ be such that $T \mu^{\prime} \cap R_{\psi} \subseteq \mu$. We show that $\left(y_{0}, y_{0}\right) \in \operatorname{int}_{R_{\psi}} W$. By 
minimality of $Y$ it follows that $\Delta_{Y} \subseteq \operatorname{int}_{R_{\psi}} W$, so $W \in \mathscr{U}_{Y} \cap R_{\psi}$. Suppose $\left(y_{0}, y_{0}\right) \notin$ int $_{R_{\psi}} W$. Then there is a net $y_{\lambda} \in \psi^{\leftarrow}(z), t_{\lambda} \in T$ such that $t_{\lambda}\left(y_{0}, y_{\lambda}\right) \rightarrow\left(y_{0}, y_{0}\right)$ while $\left(y_{0}, y_{\lambda}\right) \notin W$. Then $t_{\lambda}\left(y_{0}, y_{\lambda}\right) \in \mu^{\prime}$ eventually so $\left(y_{0}, y_{\lambda}\right) \in \mu$ eventually. But then $y_{\lambda} \in \mu\left(y_{0}\right) \subseteq V$, so $\left(y_{0}, y_{\lambda}\right) \in V \times$ $V \subseteq W$. This contradicts the choice of $y_{\lambda}$.

The following is a relativized version of Theorem 3 of [SS 74]. As seen in $\$ 5$, the condition that $Z$ be minimal cannot be dropped (or even weakened to $Z$ having a trivial action).

2.5. THEOREM. Let $Z$ be a minimal flow (a finite union of minimal flows suffices), let $\psi: Y \rightarrow Z$ be an almost periodic extension such that $\psi^{\leftarrow}(z)$ is locally connected for some $z \in Z$ and let $\phi: X \rightarrow Y$ be an open $N$-to-one extension. Then $\psi \circ \phi: X \rightarrow X$ is almost periodic. ( $X$ and $Y$ are not assumed to be minimal!).

Proof. Let $\alpha \in \mathscr{U}_{X}$ be as in Lemma 1.5, let $\gamma \in \mathscr{U}_{X}$ be open with $\bar{\gamma} \subseteq \alpha$ and let $\beta \in \mathscr{U}_{Y}$ be for $\alpha$ and $\gamma$ as in Lemma 1.5. By almost periodicity of $\psi$ there is a $\nu \in \mathscr{U}_{Y}$ with $T \nu \cap R_{\psi} \subseteq \beta$. Let $y \in \psi^{\leftarrow}(z)$ be arbitrary. Since $\phi$ is open and $N$-to-one we can find pairwise disjoint open neighbourhoods $\tilde{U}_{x^{i}}$ of $x^{i}$ in $X$ and a neighbourhood $\tilde{U}_{y}$ of $y$ in $Y$ such that $\left.\phi\right|_{\tilde{U}_{x i}}: \tilde{U}_{x_{i}} \rightarrow \tilde{U}_{y}$ is a homemorphism for $i \in\{1, \ldots, N\}$, where $\phi^{\leftarrow}(y)=\left\{x^{i} \mid 1 \leq i \leq N\right\}$. Choose a neighbourhood $U_{y}$ of $y$ with $U_{y} \subseteq \tilde{U}_{y}$ and $U_{Y} \times U_{y} \subseteq \nu$. Since $\psi(z)$ is locally connected we can find a connected neighbourhood $V_{y}$ of $y$ in $\psi^{\leftarrow}(z)$ with $V_{y} \subseteq U_{y} \cap \psi^{\leftarrow}(z)$. By compactness of $\psi^{\leftarrow}(z)$, there are finitely many $y_{1}, \ldots, y_{k}$ in $\psi^{\leftarrow}(z)$ such that $\bigcup\left\{V_{y_{j}} \mid i=1, \ldots, k\right\}=\psi^{\leftarrow}(z)$. Since $V_{y_{j}} \subseteq U_{y_{j}} \subseteq \tilde{U}_{y_{j}}$ we can find (using $\left.\phi\right|_{\tilde{U}_{x_{j}}}$ ) connected sets $V_{j}^{i} \subseteq(\psi \circ \phi)^{\leftarrow}(z)$ with $x_{j}^{i} \in V_{j}^{i} \simeq V_{y_{j}}$, where $\phi^{\leftarrow}\left(y_{j}\right)=\left\{x_{j}^{i} \mid 1 \leq i \leq N\right\}$.

(i) Define $W_{j}:=\overline{T\left(V_{y_{j}} \times V_{y_{j}}\right)} \subseteq R_{\psi}$ and $W:=\bigcup\left\{W_{j} \mid j=1, \ldots, k\right\}$. Then, by Lemma $2.4, W$ is a closed and $T$-invariant element of $R_{\psi} \cap \mathscr{U}_{Y}$.

(ii) We claim that $(\phi \times \phi) \leftarrow W_{j}=\bigcup\left\{\overline{T\left(V_{j}^{i} \times V_{j}^{L}\right)} \mid i, L=1, \ldots, N\right\}$. Obviously, $(\phi \times \phi) \leftarrow T\left(V_{y_{j}} \times V_{y_{j}}\right)=\bigcup\left\{T\left(V_{j}^{i} \times V_{j}^{L}\right) \mid i, L=1, \ldots, N\right\}$. Let $\left(x_{1}, x_{2}\right) \in(\phi \times \phi) \leftarrow W_{j}$ and let $O$ be a neighbourhood of $\left(x_{1}, x_{2}\right)$ in $R_{\psi \circ \phi}$. Since $\phi \times \phi: R_{\psi \circ \phi} \rightarrow R_{\psi}$ is open, $\phi \times \phi[O] \cap T\left(V_{y_{j}} \times V_{y_{j}}\right) \neq \phi$. So $O \cap\left\{T\left(V_{j}^{i} \times V_{j}^{L}\right) \mid i, L=1, \ldots, N\right\} \neq \phi$ and

$$
\begin{aligned}
\left(x_{1}, x_{2}\right) & \in \mathrm{cl}_{R_{\psi} \circ \phi} \bigcup\left\{T\left(V_{j}^{i} \times V_{j}^{L}\right) \mid i, L=1, \ldots, N\right\} \\
& =\bigcup\left\{\overline{T\left(V_{j}^{i} \times V_{j}^{L}\right)} \mid i, L=1, \ldots, N\right\} .
\end{aligned}
$$


Thus we have " $\subseteq$ "; the containment " $\supseteq$ " is trivial. Denote $\overline{T\left(V_{j}^{i} \times V_{j}^{L}\right)}$ by $P_{j}(i, L)$.

By (i) and (ii),

$$
\begin{aligned}
& (\phi \times \phi) \leftarrow W \\
& \quad=\bigcup\left\{P_{j}(i, L) \mid j=1, \ldots, k ; i, L=1, \ldots, N\right\} \in \mathscr{U}_{X} \cap R_{\psi \circ \phi} .
\end{aligned}
$$

So there is a $\gamma^{\prime} \in \mathscr{U}_{X}$ with $\gamma^{\prime} \subseteq \gamma$ such that

$$
\gamma^{\prime} \cap R_{\psi \circ \phi} \subseteq \bigcup\left\{P_{j}(i, L) \mid j=1, \ldots, k ; i, L=1, \ldots, N\right\} .
$$

Let $F=\left\{(j, i, L) \mid \gamma^{\prime} \cap P_{j}(i, L) \neq \varnothing\right\}$ and for $f=(j, i, L) \in F$ denote $P_{f}:=P_{j}(i, L)$. Then $\gamma^{\prime} \cap R_{\psi \circ \phi} \subseteq \bigcup\left\{P_{f} \mid f \in F\right\}$. Since $P_{f}$ is closed and $T$-invariant and $F$ is finite, it follows that

(iii) $\overline{T \gamma^{\prime} \cap R_{\psi \circ \phi}} \subseteq \cup\left\{P_{f} \mid f \in F\right\}$. We claim that

(iv) $P_{f} \subseteq T \bar{\gamma} \cap R_{\psi \circ \phi}$ for every $f \in F$. Indeed, let $f=(j, i, L) \in F$. Then $\varnothing \neq \gamma^{\prime} \cap P_{f} \subseteq \gamma \cap P_{f}$, say $t_{f}\left(\bar{x}_{1}, \bar{x}_{2}\right) \in \gamma$ for some $t_{f} \in T,\left(\bar{x}_{1}, \bar{x}_{2}\right)$ $\in V_{j}^{i} \times V_{j}^{L}$. As $t_{f}\left(\bar{x}_{1}, \bar{x}_{2}\right)$ is an almost periodic point, there is a (discretely) syndetic set $B_{f} \subseteq T$ with $B_{f} t_{f}\left(\bar{x}_{1}, \bar{x}_{2}\right) \subseteq \gamma$. Let $A_{f}$ be finite with $T=A_{f} B_{f}=A_{f} B_{f} t_{f}$. For every $b \in B_{f}, b t_{f}\left(\bar{x}_{1}, \bar{x}_{2}\right) \in b t_{f}\left(V_{j}^{i} \times V_{j}^{L}\right) \cap \gamma$ and, obviously, $b t_{f}\left(V_{j}^{i} \times V_{j}^{L}\right)$ is connected. Since

$$
\begin{aligned}
& \phi \times \phi\left[b t_{f}\left(V_{j}^{i} \times V_{j}^{L}\right)\right] \\
& \quad=b t_{f}\left(V_{y_{j}} \times V_{y_{j}}\right) \subseteq b t_{f}\left(U_{y_{j}} \times U_{y_{j}}\right) \cap R_{\psi} \subseteq T \nu \cap R_{\psi} \subseteq \beta
\end{aligned}
$$

it follows from Lemma 1.5 that $b t_{f}\left(V_{j}^{i} \times V_{j}^{L}\right) \subseteq \gamma \cup \alpha^{c}$. Hence, by connectedness, $b t_{f}\left(V_{j}^{i} \times V_{j}^{L}\right) \subseteq \gamma$. So, since $b \in B_{f}$ was arbitrary, $B_{f} t_{f}\left(V_{j}^{i} \times V_{j}^{L}\right) \subseteq \gamma$. But then

$$
T\left(V_{j}^{i} \times V_{j}^{L}\right)=A_{f} B_{f} t_{f}\left(V_{j}^{i} \times V_{j}^{L}\right) \subseteq A_{f} \gamma .
$$

Hence $P_{f}=\overline{T\left(V_{j}^{i} \times V_{j}^{L}\right)} \subseteq \overline{A_{f} \gamma}=A_{f} \bar{\gamma} \subseteq T \bar{\gamma}$, which proves our claim. By (ii) and (iv) it follows that

$$
\overline{T \gamma^{\prime} \cap R_{\psi \circ \phi}} \subseteq \bigcup\left\{P_{f} \mid f \in F\right\} \subseteq T \bar{\gamma} \cap R_{\psi \circ \phi} .
$$

Since $Q_{\psi \circ \phi} \subseteq \overline{T \gamma^{\prime} \cap R_{\psi \circ \phi}}$ and $\gamma$ can be chosen arbitrarily small, we have $Q_{\psi \circ \phi} \subseteq P_{\psi \circ \phi}$. However, $\psi \circ \phi$ is distal, so $P_{\psi \circ \phi}=\Delta_{X}$ and it follows that $Q_{\psi \circ \phi}=\Delta_{X}$. This shows that $\psi \circ \phi$ is almost periodic.

2.6. Corollary. Let $Z$ be a minimal flow, let $\theta: W \rightarrow Z$ be an open $N$-to-one extension, $\xi: Y \rightarrow W$ an almost periodic extension with locally connected fibers and let $\phi: X \rightarrow Y$ be a quotient group extension with totally disconnected fibers. Then $\theta \circ \xi \circ \phi: X \rightarrow Z$ is an almost periodic extension. 
Proof. By $1.3, \psi=\theta \circ \xi$ is an almost periodic extension, and obviously, $\psi$ has a locally connected fiber. By $1.4, \phi$ is the inverse limit of open finite-to-one extension $\phi_{N}: X_{N} \rightarrow Y$. From 2.5 we know that $\psi \circ \phi_{N}$ is almost periodic, hence $\psi \circ \phi$ is almost periodic.

3. The minimal case. In this section we confine ourselves to extensions of minimal flows and we prove the relativized version of 2.2 (3.2). In doing so we need the characterization of the regionally proximal relation for distal extensions of minima flows as stated in 2.6.1 of [V 77].

The proof uses $\mathscr{F}$-topology techniques.

For details about $\mathscr{F}$-topologies we refer to [V 77] or [Wo 82] Ch. III, VIII.

In the following $\mathscr{U}_{x}$ denotes the neighbourhood filter of $x$ in $X$, and $u$ is an idempotent in $M$, the universal minimal flow for $T$. In the next lemma we use the same technique used in 2.2 .

3.1. LeMma. Let $\phi: X \rightarrow Y$ be an open $N$-to-one extension of minimal flows. Let $\psi: Y \rightarrow Z$ be almost periodic with a connected fiber $\psi^{\leftarrow}(z)$ and let $x=u x \in \phi^{\leftarrow} \psi^{\leftarrow}(z)$. Then for $\gamma \in \mathscr{U}_{X}$ small enough there is an open neighbourhood $U$ of $u$ in $M$ such that for $B(U)=\{t \mid t u \in U\} \subseteq T$ we have $B(U) \cdot\left(\gamma \cap \phi^{\leftarrow} \psi^{\leftarrow}(z) \times \phi^{\leftarrow} \psi^{\leftarrow}(z)\right) \subseteq \gamma^{3}$.

Proof. Let $x \mapsto y \mapsto z, \alpha \in \mathscr{U}_{X}$ as in Lemma 1.5; $\gamma=\gamma^{-1} \in \mathscr{U}_{X}$ with $\gamma \subseteq \bar{\gamma} \subseteq \alpha$. Let $\beta \in \mathscr{U}_{Y}$ be for $\alpha$ and $\gamma$ as in Lemma 1.5. Since $\psi$ is almost periodic, there is a neighbourhood $U_{0}$ of $u$ in $M$ such that $U_{0} y^{\prime} \subseteq \beta\left(y^{\prime}\right)$ for every $y^{\prime} \in \psi^{\leftarrow}(z)$ (Lemma 2.1). Since $\psi^{\leftarrow}(z)$ is connected and $\phi$ is $N$-to-one, $\phi^{\leftarrow} \psi^{\leftarrow}(z)$ has finitely many components, say $C_{1}, \ldots, C_{m}(m \leq N)$. Choose $x_{i} \in C_{i}$, then there are open neighbourhoods $U_{i}$ of $u$ in $M$ such that $U_{i} x_{i} \subseteq \gamma\left(x_{i}\right)$. Define $U=\bigcap_{i=0}^{m} U_{i}$ and $B(U)$ as above. For every $b \in B(U)$ define

$$
A_{i}(b)=\left\{x \in C_{i} \mid(x, b x) \in \gamma\right\}, \quad \text { note that } x_{i} \in A_{i}(b) .
$$

Since $\gamma$ is open, $A_{i}(b)$ is open in $C_{i}$. Also, $A_{i}(b)$ is closed in $C_{i}$ :

Let $x \in C_{i}$ be such that $(x, b x) \in \bar{\gamma} \subseteq \alpha$. Then $\phi \times \phi(x, b x)=$ $(y, b y) \in \beta \quad\left(b u \in U_{0}\right)$. Hence, by Lemma 1.5, $(x, b x) \in \gamma \cup \alpha^{c}$, so $(x, b x) \in \gamma$. So $A_{i}(b)=\left\{x \in C_{i} \mid(x, b x) \in \bar{\gamma}\right\}$ and as $\bar{\gamma}$ is closed, $A_{i}(b)$ is closed.

Since $C_{i}$ is connected and $A_{i}(b) \neq \varnothing, a_{i}(b)=C_{i}$. It follows that $b x \in \gamma(x)$ for every $x \in \bigcup_{i=1}^{m} C_{i}=\phi^{\leftarrow} \psi^{\leftarrow}(z)$. Let $\left(x_{1}, x_{2}\right) \in \gamma \cap$ $\phi^{\leftarrow} \psi^{\leftarrow}(z) \times \phi^{\leftarrow} \psi^{\leftarrow}(z)$ and let $b \in B(U)$. Then $\left(b x_{1}, b x_{2}\right)=\left(b x_{1}, x_{1}\right) \circ$ $\left(x_{1}, x_{2}\right) \circ\left(x_{2}, b x\right) \in \gamma^{3}$. 
3.2. THEOREM. Let $\phi: X \rightarrow Y$ be an open $N$-to-one extension of minimal flows. Let $\psi: Y \rightarrow Z$ be an almost periodic extension with a connected fiber $\psi^{\leftarrow}(z)$. Then $\psi \circ \phi: X \rightarrow Z$ is almost periodic.

Proof. Let $\gamma \in \mathscr{U}_{X}$ be open and small enough. Then, by Lemma 3.1. with the same notation,

$$
B(U) \cdot\left(\gamma \cap \phi^{\leftarrow} \psi^{\leftarrow}(z) \times \phi^{\leftarrow} \psi^{\leftarrow}(z)\right) \subseteq \gamma^{3}
$$

for some open neighbourhood $U$ of $u$ in $M$. Choose $x=u x \in \phi^{\leftarrow} \psi^{\leftarrow}(z)$ and let $x^{\prime} \in Q_{\psi \circ \phi}[x]$, i.e. $\left(x, x^{\prime}\right) \in Q_{\psi \circ \phi}$. Then, by 2.6.1 of [V 77] (this is where minimality is used), there are $t_{\lambda} \in T, x_{\lambda} \in \phi^{\leftarrow} \psi^{\leftarrow}(z)$ such that

$$
t_{\lambda} \rightarrow u ; x_{\lambda} \rightarrow x \text { and } t_{\lambda} x_{\lambda} \rightarrow x^{\prime} \text {. }
$$

For a certain $\lambda_{1}$ we have $\left(x, x_{\lambda}\right) \in \gamma$ for every $\lambda \geq \lambda_{1}$. Since also $t_{\lambda} u \rightarrow u$, there is a $\lambda_{2}$ such that $t_{\lambda} u \in U$ for every $\lambda \geq \lambda_{2}$. Hence $t_{\lambda} \in B(U)$ for every $\lambda \geq \lambda_{2}$. So for every $\lambda \geq \max \left(\lambda_{1}, \lambda_{2}\right)$ we have $t_{\lambda} \in B(U)$ and $\left(x, x_{\lambda}\right) \in \gamma$. Consequently, by $(*), t_{\lambda}\left(x, x_{\lambda}\right) \in \gamma^{3}$ for every $\lambda \geq$ $\max \left(\lambda_{1}, \lambda_{2}\right)$, and so $\left(x, x^{\prime}\right)=\lim t_{\lambda}\left(x, x_{\lambda}\right) \in \overline{\gamma^{3}}$.

Since $\gamma$ may be chosen arbitrarily small, it follows that $x=x^{\prime}$; i.e. $Q_{\psi \circ \phi}[x]=\{x\}$. Since $\psi \circ \phi$ is a distal map it follows that $Q_{\psi \circ \phi}=\Delta_{X}$, which shows that $\psi \circ \phi$ is almost periodic.

3.3. Corollary. Let $\phi: X \rightarrow Y$ be an almost periodic extension of minimal flows with totally disconnected fibers and let $\psi: Y \rightarrow Z$ be an almost periodic extension with a fiber $\psi^{\leftarrow}(z)$ having finitely many components. Then $\psi \circ \phi$ is almost periodic.

Proof. Let $R c(\psi) \subseteq R_{\psi}$ be the closed invariant equivalence relation that identifies the points in the same component (cf. [MW 76] 2.3.). Then $\theta: Y / R c(\psi) \rightarrow Z$ is open with a finite fiber, hence $\theta$ is finite-to-one, and $\kappa: Y \rightarrow Y / R c(\psi)$ has connected fibers. Since an almost periodic extension of minimal flows is a quotient group extension (e.g. [MW 76] 1.1) it follows that 1.4 that $\phi$ is the inverse limit of finite-to-one extensions. But then, by 3.5 and an inverse limit procedure it follows that $\kappa \circ \phi$ is almost periodic. So, by $1.3, \psi \circ \phi$ is almost periodic.

Note that 3.3 also covers the case where some fiber of $\psi$ is locally connected. For a locally connected compact $T_{2}$ space has a finite number of components (compare 2.5).

Let $\phi: X \rightarrow Y$ be a distal extension of minimal flows. In [MW 76] 3.7, modulo the non-metric version of the Furstenberg structure theorem [MW 81], it was shown that the quotient map $X \rightarrow X / E_{\phi}$ has connected 
fibers, as long as the phase group is semi-compactly generated. In 3.4 we replace the group assumption by the assumption of $X / E_{\phi} \rightarrow Y$ having a fiber with finitely many components. From this it follows that $\phi$ is connected iff $X / E_{\phi} \rightarrow Y$ is connected.

3.4. THEOREM. Let $\phi: X \rightarrow Y$ be a distal extension of minimal flows. If $\theta: X / E_{\phi} \rightarrow Y$ has a fiber $\theta^{\leftarrow}(y)$ with finitely many components, then $\kappa:$ $X \rightarrow X / E_{\phi}$ has connected fibers. In particular, $\phi: X \rightarrow Y$ has a fiber with the same amount of components as $\theta^{\leftarrow}(y)$.

Proof. Let $R c(\theta)$ be the connectedness structure relation for $\theta$ and let $Z=X_{1} / R c(\theta)$ where $X_{1}=X / E_{\phi}$. Then, as in the proof of $3.3 Z \rightarrow Y$ is an open $N$-to-one extension ( $N$ is the amount of components in $\theta^{\leftarrow}(y)$ ), and $X_{1} \rightarrow Z$ has connected fibers. Since $\phi: X \rightarrow Z$ is distal, it follows from the Furstenberg structure theorem that there exists a tower $\left\{\phi_{\beta}^{\alpha}\right.$ : $X_{\alpha} \rightarrow X_{\beta} \mid 0 \leq \beta \leq \alpha \leq \nu$ ) of height $\nu$ with $X_{0}=Z, X_{1}=X / E_{\phi}, X_{\nu}=X$ and $\phi_{\alpha}^{\alpha+1}: X_{\alpha+1} \rightarrow X_{\alpha}$ is almost periodic. Moreover, if $\phi_{\alpha}: X \rightarrow X_{\alpha}$, $X_{\alpha+1}=X / E_{\phi_{\alpha}}$, so $\phi_{\alpha}^{\alpha+1}$ is the maximal almost periodic factor of $\phi_{\alpha}$.

By transfinite induction we show that $\phi_{0}^{\alpha}: X_{\alpha} \rightarrow X_{0}$ has connected fibers for every $\alpha \leq \nu$. We know already that $\phi_{0}^{1}$ has connected fibers. Let $\alpha$ be an ordinal and suppose $\phi_{0}^{\beta}: X_{\beta} \rightarrow X_{0}$ has connected fibers for every $\beta<\alpha$. If $\alpha$ is a limit ordinal clearly $\phi_{0}^{\alpha}=\operatorname{inv} \lim \left\{\phi_{0}^{\beta} \mid \beta<\alpha\right\}$ has connected fibers. If $\alpha$ is not a limit ordinal then $\phi_{0}^{\alpha-1}: X_{\alpha-1} \rightarrow X_{0}$ has connected fibers. Since $\phi_{\alpha-1}^{\alpha}$ is almost periodic, the induced map $\left(\phi_{\alpha-1}^{\alpha}\right)_{c}$ : $X_{\alpha} / R c\left(\phi_{0}^{\alpha}\right) \rightarrow X_{\alpha-1} / R c\left(\phi_{0}^{\alpha-1}\right)$ is almost periodic ([MW 76] 3.7). But $X_{\alpha-1} / R c\left(\phi_{0}^{\alpha-1}\right) \simeq X_{0}$, so $X_{1}$ factorizes over $X_{\alpha} / R c\left(\phi_{0}^{\alpha}\right)$. Hence, $\left(\phi_{\alpha-1}^{\alpha}\right)_{c}$ has connected fibers. Since $X_{\alpha} \rightarrow X_{\alpha} / R c\left(\phi_{0}^{\alpha}\right)$ has connected fibers, so has $\phi_{0}^{\alpha}$. Consequently, $\phi_{1}^{\nu}: X \rightarrow X_{1}=X / E_{\phi}$ has connected fibers. Moreover, $\phi_{0}^{\nu}: X \rightarrow Z$ has connected fibers so $\phi$ has a fiber with $N$ components.

4. Local almost periodicity. In the previous sections we have seen that under certain connectedness conditions on an almost periodic extension $\psi$, the extension $\psi \circ \phi$ is almost periodic for an open $N$-to-one extensions $\phi$. It is only natural to ask whether or not something similar holds for a locally almost periodic extension $\psi$.

Example $\$ 6$ shows that just connectedness for $\psi$ is not enough, neither is the assumption of the phase group being semi-compactly generated. But it will turn out that (in the minimal case) locally connectedness for $\psi$ suffices.

Recall that a map $\eta: X \rightarrow Y$ is highly proximal iff $\eta$ is irreducible $\left(\eta[\bar{A}]=Y\right.$ implies $\bar{A}=X$ ) iff $t_{\imath} \eta^{\leftarrow}(y)$ converges to a singleton in $2^{X}$ for a certain net $\left\{t_{i}\right\}$ in $T$, in particular, $u \circ \eta^{\leftarrow}(y)=\{u x\}$ for every 
$x \in \eta^{\leftarrow}(y)$. An extension $\phi: X \rightarrow Z$ of minimal flows is locally almost periodic iff $\phi=\xi \circ \eta$ where $\xi: Y \rightarrow Z$ almost periodic and $\eta: X \rightarrow Y$ is highly proximal [MW 80].

For details concerning highly proximal extensions we refer to [AG 77], [AW 81] and [Wo 82] Ch. IV, and for local almost periodicity (of maps) to [MW 72] and [MW 80], (Note the terminology of [MW 80] is "almost equicontinuous" instead of "locally almost periodic", 1.12 and 1.15 if [MW 80] yield the above characterization).

First we are concerned with interchanging an open $\mathrm{N}$-to-one extension and a highly proximal extension.

4.1. THEOREM. Let $\phi: X \rightarrow Y$ be an open $N$-to-one extension of minimal flows and let $\psi: Y \rightarrow Z$ be highly proximal with connected fibers. Then $\psi \circ \phi=\xi \circ \eta$ where $\eta: X \rightarrow X / R c(\psi \circ \phi)$ is highly proximal and $\xi$ : $X / R c(\psi \circ \phi) \rightarrow Z$ is open and $N$-to-one.

Proof. First we show that for every $z \in Z$ the fiber $(\psi \circ \phi)^{\leftarrow}(z)$ consists of $N$ components and that points in different components are distal:

Let $y=u \circ \psi^{\leftarrow}(z)$. Since $\phi$ is $N$-to-one and open, we can choose an open neighbourhood $U_{y}$ of $y$ and open neighbourhoods $U_{x} i$ such that $U_{x} i \simeq U_{y}$, for every $i \in\{1, \ldots, N\}$. Then for some $t$ close to $u$ in $S_{T}$ we have $t \psi^{\leftarrow}(z) \subseteq U_{y}$, and note that $t \psi^{\leftarrow}(z)=\psi^{\leftarrow}(t z)$ is connected.

Define $C_{i}=t^{-1}\left(U_{x} i \cap \phi^{\leftarrow} x^{\leftarrow}(t z)\right)$. Then $\phi^{\leftarrow} \psi^{\leftarrow}(t z)=\bigcup\left\{t C_{i} \mid i=\right.$ $1, \ldots, N\}$ and, since $\left.\phi\right|_{U_{x} i}$ is a homeomorphism, every $t C_{i}$ is connected. Since the $U_{x} i$ can be chosen such that their closures are disjoint, the $t C_{i}$ 's are just the components of $(\psi \circ \phi)^{\leftarrow}(t z)$. Hence $\left\{C_{i} \mid i=1, \ldots, N\right\}$ are the components of $(\psi \circ \phi) \leftarrow(z)$. Note that $\phi\left[C_{i}\right]=\psi^{\leftarrow}(z)$ for every $i \in\{1, \ldots, N\}$.

Let $x_{1} \in C_{1}$ and $x_{2} \in C_{2}$, choose $x_{2}^{\prime} \in C_{2}$ such that $\phi\left(x_{1}\right)=\phi\left(x_{2}^{\prime}\right)$. Then $\left(x_{1}, x_{2}^{\prime}\right)$ is a distal pair. Suppose $\left(x_{1}, x_{2}\right) \in P_{X}$ then there is a minimal left ideal $I$ and a $v \in I$ with $v x_{1}=v x_{2}$. Since $v \circ \psi^{\leftarrow}(z)=\bar{y}$ for some $\bar{y} \in Y, v \circ \bigcup_{i=1}^{n} C_{i}=\phi^{\leftarrow} \bar{y}$ ( $\phi$ is open!). So every $v \circ C_{i}$ is a point ( $v \circ C_{i}$ is connected!)

Hence $v x_{2}=v x_{2}^{\prime}$; but then $v x_{1}=v x_{2}=v x_{2}^{\prime}$, and $x_{1}=x_{2}^{\prime}$ which contradicts the fact that $C_{1} \cap C_{2}=\varnothing$.

Define $\eta: X \rightarrow X / R c(\psi \circ \phi)$, then $\eta$ identifies exactly the points in each component. By $(*)$ it follows that $\eta$ is highly proximal. Let $\xi$ : $X / R c(\psi \circ \phi) \rightarrow Z$; then, obviously, $\xi$ is an $N$-to-one extension. Also $\xi$ is 
distal (and so open), since any pair of proximal points in $X / R C(\psi \circ \phi)$, comes from a pair of proximal points in $X$ which must be in the same connected component by the above.

As the technicalities in the proof of 4.4 obscure the basic idea, we will ilustrate that basic idea by first proving an absolute version of 4.4 that is slightly stronger. Note it is easy to see that if $X$ is proximal and locally connected then it is connected.

4.2. TheOREM. Let $\phi: X \rightarrow Y$ be a proximal homomorphism and let $Y$ be almost periodic. If $X$ is locally connected then $\phi$ has connected fibers. ( $X$ not necessarily minimal.)

Proof. Suppose $\phi^{\leftarrow}(y)$ is not connected. Then there are open sets $U_{1}$ and $U_{2}$ in $X$ such that $U_{1} \cap U_{2}=\varnothing$ and $\phi^{\leftarrow}(y) \subseteq U_{1} \cup U_{2}$. Choose $x_{i} \in \phi^{\leftarrow}(y) \cap U_{i}$ and let $(w, w) \in \overline{T\left(x_{1}, x_{2}\right)}$ ( $\phi$ is proximal!) Let $\left\{W_{\lambda} \mid \lambda\right.$ $\in \Lambda\}$ be a neighbourhood filter base at $w$ consisting of connected open sets. Then there are $t_{\lambda} \in T$ with $t_{\lambda}\left(x_{1}, x_{2}\right) \in W_{\lambda} \times W_{\lambda}$. Since $t_{\lambda}^{-1} W_{\lambda}$ is connected and $t_{\lambda}^{-1} W_{\lambda} \cap U_{i} \neq \varnothing$ it follows that $t_{\lambda}^{-1} W_{\lambda} \backslash\left(U_{1} \cup U_{2}\right) \neq \varnothing$. Choose $w_{\lambda} \in W_{\lambda}$ such that $v_{\lambda}:=t_{\lambda}^{-1} w_{\lambda} \in t_{\lambda}^{-1} W_{\lambda} \backslash\left(U_{1} \cup U_{2}\right)$.

Then $v_{\lambda} \rightarrow v \in U_{1} \cup U_{2}$ while $w_{\lambda} \rightarrow w$. Hence

$$
t_{\lambda}\left(v_{\lambda}, x_{1}\right) \rightarrow(w, w) \text { and }\left(v_{\lambda}, x_{1}\right) \rightarrow\left(v, x_{1}\right) .
$$

This shows that $v \in Q_{X}\left[x_{1}\right]$. Since $P_{X}=O_{X}=R_{\phi}$, it follows that $v \in \phi^{\leftarrow}$ (y). But then $v \in U_{1} \cup U_{2}$, which was not the case. This contradiction shows that $\phi^{\leftarrow}(y)$ is connected.

Before we can show that the highly proximal extension that is part of an open locally connected locally almost periodic extension has connected fibers we need the following lemma.

4.3. LeMma. Let $\theta: X \rightarrow Z$ be an open locally almost periodic extension of minimal flows, say $\theta=\psi \circ \phi, \phi: X \rightarrow Y$ highly proximal and $\psi: Y \rightarrow Z$ almost periodic. Let $z \in Z$ and $W \subseteq(\psi \circ \phi)^{\leftarrow}(z)$ such that $\phi[W]$ has a nonempty interior in $\psi^{\leftarrow}(z)$. Then $\overline{T\left(W \times W \cap R_{\psi \circ \phi}\right)} \in \mathscr{U}_{X} \cap R_{\psi \circ \phi}$.

Proof. Since $\phi[W]^{\circ}$ is open in $\psi^{\leftarrow}(z)$ and $\psi$ is almost periodic, it follows from 2.4 (minimal case) that $\overline{T\left(\phi[W] \times \phi[W] \cap R_{\psi}\right)} \in \mathscr{U}_{Y} \cap$ $R_{\psi}$. Suppose $(x, x) \notin \operatorname{int}_{R_{\psi \circ \phi}} \overline{T\left(W \times W \cap R_{\psi \circ \phi}\right)}$. Then for every neighbourhood $O$ of $(x, x)$ in $R_{\psi \circ \phi}, O^{\prime}=O \backslash \frac{T\left(W \times W \cap R_{\psi \circ \phi}\right)}{T(s}$ nonempty and open. 
By [Wo 82] IV. 4.13 (with $\phi=\psi, \sigma=\xi, \tau=\mathrm{id}, \phi^{\prime}$ referring to our $\theta$ ), $\phi \times \phi: R_{\psi \circ \phi} \rightarrow R_{\psi}$ is an irreducible surjection. So there is an open set $P_{O}=P$ in $R_{\psi \circ \phi}$ such that $P=\left(\phi \times\left.\phi\right|_{R_{\psi \circ \phi}}\right)^{\leftarrow} \phi \times \phi[P]$ and $P \subseteq O^{\prime}$. For every $O$ choose $\left(x_{0}^{1}, x_{0}^{2}\right) \in P_{O}$, then $\left(x_{0}^{1}, x_{0}^{2}\right) \rightarrow(x, x)$. So $\left(\phi\left(x_{0}^{1}\right), \phi\left(x_{0}^{2}\right)\right) \rightarrow(\phi(x), \phi(x))$, and since $\overline{T\left(\phi[W] \times \phi[W] \cap R_{\psi}\right)} \in \mathscr{U}_{Y}$ $\cap R_{\psi}$,

$\left(\phi\left(x_{0}^{1}\right), \phi\left(x_{0}^{2}\right)\right) \in \overline{T\left(\phi[W] \times \phi[W] \cap R_{\psi}\right)}=\phi \times \phi \overline{T\left(W \times W \cap R_{\psi \circ \phi}\right)}$ eventually. But $P_{O}$ consisted of full fibers under $\phi \times\left.\phi\right|_{R_{\psi} \circ \phi}$ and $P_{O} \cap$ $\overline{T\left(W \times W \cap R_{\psi \circ \phi}\right)}=\varnothing$, hence

$$
\left(\phi\left(x_{0}^{1}\right), \phi\left(x_{0}^{2}\right)\right) \notin \overline{T\left(\phi[W] \times \phi[W] \cap R_{\psi}\right)} .
$$

This contradiction shows that $(x, x) \in \operatorname{int}_{R_{\psi \circ \phi}} \overline{T\left(W \times W \cap R_{\psi \circ \phi}\right)}$. Since $x$ was arbitrary it follows that $\Delta_{X} \subseteq \operatorname{int}_{R_{\psi \circ \phi}} \frac{\phi\left(W \times W \cap R_{\psi \circ \phi}\right)}{T(W .}$

4.4. THEOREM. Let $\phi: X \rightarrow Y$ be a highly proximal extension of minimal sets and let $\psi: Y \rightarrow Z$ be almost periodic such that $\psi \circ \phi$ is open and has a locally connected fiber. Then $\phi$ has connected fibers.

Proof. Let $y \in Y$ and suppose $\phi^{\leftarrow}(y)$ is not connected. Then there are open sets $U_{1}, U_{2}$ with $\phi^{\leftarrow}(y) \subseteq U_{1} \cup U_{2}, U_{i} \cap \phi^{\leftarrow}(y) \neq \varnothing$ and $U_{1} \cap U_{2}=\varnothing$. Consider a base $\mathscr{B}$ for $\mathscr{U}_{X}$ ordered by inclusion. For every $\mu \in \mathscr{B}$ construct open sets $U_{i}^{\mu} \subseteq U_{i}$ such that $\phi^{\leftarrow}(y) \cap U_{i}^{\mu} \neq \varnothing$ and $\phi^{\leftarrow}(y)=\bigcap\left\{U_{1}^{\mu} \cup U_{2}^{\mu} \mid \mu \in \mathscr{U}_{X}\right\}$.

Let $z \in Z$ be such that $(\psi \circ \phi) \leftarrow(z)$ is locally connected. For $\mu \in \mathscr{U}_{X}$ and $x \in(\psi \circ \phi)^{\leftarrow}(z)$ choose connected closed neighbourhoods $W_{x}^{u}$ of $x$ in $(\psi \circ \phi)^{\leftarrow}(z)$, with $W_{x}^{\mu} \subseteq \mu(x)$. Then there is a finite collection $\left\{W_{x_{i}}^{\mu} \mid i\right.$ $\left.=1, \ldots, n_{\mu}\right\}$ such that

$$
(\psi \circ \phi)^{\leftarrow}(z)=\bigcup\left\{W_{x_{i}}^{\mu} \mid i=1, \ldots, n_{\mu}\right\} .
$$

Since $\psi^{\leftarrow}(z)=\bigcup\left\{\phi\left[W_{x_{i}}^{\mu}\right] \mid i=1, \ldots, n_{\mu}\right\}$, there is an $i_{\mu} \in\left\{1, \ldots, n_{\mu}\right\}$ with $\phi\left[W_{x_{i}}^{\mu}\right]^{\circ} \neq \varnothing$ in $\psi^{\leftarrow}(z)$. Define $W^{\mu}:=W_{x_{i_{i}}}^{\mu}$. Take a subnet of $\mathscr{B}$ such that $x_{i_{\mu}} \rightarrow \bar{x} \in(\psi \circ \phi)^{\leftarrow}(z)$, and note that if $w_{\mu} \in W^{\mu}$ then $w_{\mu} \rightarrow \bar{x}$ in $(\psi \circ \phi) \leftarrow(z)$. By Lemma 4.3, $\overline{T\left(W^{\mu} \times W^{\mu} \cap R_{\psi \circ \phi}\right)} \in \mathscr{U}_{X} \cap R_{\psi \circ \phi}$. Note that proximality of $\phi$ and the fact that $U_{i}^{\mu} \cap \phi^{\leftarrow}(y) \neq \varnothing$ imply that $\Delta_{X} \subseteq \overline{T\left(U_{1}^{\mu} \times U_{2}^{\mu} \cap R_{\psi \circ \phi}\right)}$. Hence

$$
\overline{T\left(W^{\mu} \times W^{\mu} \cap R_{\psi \circ \phi}\right)} \cap U_{1}^{\mu} \times U_{2}^{\mu} \cap R_{\psi \circ \phi} \neq \varnothing .
$$


So, by openness of $U_{1}^{\mu} \times U_{2}^{\mu} \cap R_{\psi \circ \phi}$, it follows that there is a $t_{\mu}$ with

$$
t_{\mu}\left(W^{\mu} \times W^{\mu}\right) \cap U_{1}^{\mu} \times U_{2}^{\mu} \cap R_{\psi \circ \phi} \neq \varnothing .
$$

Say $t_{\mu}\left(w_{1}^{\mu}, w_{2}^{\mu}\right) \in U_{1}^{\mu} \times U_{2}^{\mu} \cap R_{\psi \circ \phi}$. Then, after passing to a suitable subnet, $t_{\mu}\left(w_{1}^{\mu}, w_{2}^{\mu}\right) \rightarrow\left(\bar{x}_{1}, \bar{x}_{2}\right)$; where $\bar{x}_{i} \in \phi^{\leftarrow}(y)$, and $\left(w_{1}^{\mu}, w_{2}^{\mu}\right) \rightarrow(\bar{x}, \bar{x})$. Since $t_{\mu} W^{\mu}$ is connected and $\varnothing \neq t_{\mu} W^{\mu} \cap U_{i}^{\mu} \subseteq t_{\mu} W^{\mu} \cap U_{i}$ it follows that $t_{\mu} W^{\mu} \nsubseteq U_{1} \cup U_{2}$; say $t_{\mu} v^{\mu} \in t_{\mu} W^{\mu} \backslash U_{1} \cup U_{2}$ for some $v^{\mu} \in W^{\mu}$. Let $t_{\mu} v^{\mu} \rightarrow v$ and note that $v^{\mu} \rightarrow \bar{x}$. Also, note that $v \notin U_{1} \cup U_{2}$. But then $t_{\mu}\left(v^{\mu}, w_{1}^{\mu}\right) \rightarrow\left(v, \bar{x}_{1}\right)$, while $\left(v^{\mu}, w_{1}^{\mu}\right) \rightarrow(\bar{x}, \bar{x})$. So $\left(v, \bar{x}_{1}\right) \in Q_{\psi \circ \phi} ;$ but, since $\psi \circ \phi$ is locally almost periodic, $Q_{\psi \circ \phi}=P_{\psi \circ \phi}=P_{\phi}$. Hence $\left(v, \bar{x}_{1}\right) \in P_{\phi}$, so $v \in \phi^{\leftarrow}(y) \subseteq U_{1} \cup U_{2}$ while $v \notin U_{1} \cup U_{2}$. This is a contradiction, showing that $\phi^{\leftarrow}(y)$ is connected.

4.5. TheOREM. Let $\phi: X \rightarrow Y$ be an open $N$-to-one extension of minimal flows. Let $\psi: Y \rightarrow Z$ be open and locally almost periodic with a locally connected fiber. Then $\psi \circ \phi$ is locally almost periodic.

Proof. Let $\psi: Y \rightarrow Z$ be given by $\eta: Y \rightarrow W$ highly proximal and $\xi$ : $W \rightarrow Z$ almost periodic. By Theorem 4.4, $\eta$ has connected fibers. Then, by Lemma $4.1, n \circ \phi=\alpha \circ \beta$, where $\beta$ is highly proximal and $\alpha$ is open and $N$-to-one. So $\xi \circ \alpha$ is almost periodic by 2.5. Since $\psi \circ \phi=\xi \circ \eta \circ \phi=$ $\xi \circ \alpha \circ \beta$, it follows that $\psi \circ \phi$ is locally almost periodic.

4.6. Corollary. Let $\phi: X \rightarrow Y$ be an almost periodic extension of minimal flows with totally disconnected fibers and let $\psi: Y \rightarrow Z$ be locally almost periodic with locally connected fibers, then $\psi \circ \phi$ is locally almost periodic.

Proof. After noting that an inverse limit of locally almost periodic extensions is locally almost periodic again, this follows in the same way as 3.6.

5. Example related to 2.2 and 2.5. We give an example showing that 2.2 cannot be relativized without further restrictions on the "smallest" flow $Z(\{*\}$ in 2.2.) and that 2.5 is not true without the minimality condition on $Z$ (even $Z$ semisimple is not sufficient).

We were unable to prove or disprove the relativized case for 2.2 where $Z$ is minimal.

We construct an open 2-to-one extension $\phi: X \rightarrow Y$ and an almost periodic homomorphism $\psi: Y \rightarrow Z$ with connected locally connected fibers, such that $\psi \circ \phi: X \rightarrow Z$ is not almost periodic. 
Define $Z=\{0\} \cup\{1 / n \mid n \in \mathbf{N}\}$ with a trivial action, so $Z$ is semisimple. Define

$$
X=Y=\left\{\left(\frac{1}{n}, \frac{\sin \theta}{n}, \cos \theta\right) \mid n \in \mathbf{N}, \theta \in \mathbf{R}\right\} \cup\{(0,0, \cos \theta) \mid \theta \in \mathbf{R}\} .
$$

So $X=Y$ is a collection of ellipses with decreasing small axes, converging to an interval.

For each $n \in \mathbf{N}$ define homeomorphisms $f_{n}, g_{n}$ and $h_{n}$ on $X, Y$ and $Z$ by

$$
\begin{aligned}
& \begin{array}{l}
f_{n}\left(\left(\frac{1}{n}, \frac{\sin \theta}{n}, \cos \theta\right)\right)=\left(\frac{1}{n}, \frac{-\sin (\theta+\pi / 2)}{n}, \cos (\theta+\pi / 2)\right) \\
\text { and } f_{n} \text { leaves the rest of } X \text { fixed; }
\end{array} \\
& \begin{aligned}
g_{n}\left(\left(\frac{1}{n}, \frac{\sin \theta}{n}, \cos \theta\right)\right)= & \left(\frac{1}{n}, \frac{\sin \theta}{n},-\cos \theta\right) \\
& \text { and } g_{n} \text { leaves the rest of } Y \text { fixed; }
\end{aligned} \\
& h_{n} \text { leaves all of } Z \text { fixed. }
\end{aligned}
$$

Let $T$ be the free group generated by $\mathbf{N}$ and define the action of $T$ on $X, Y$ and $Z$ by the homeomorphisms $f_{n}, g_{n}$ and $h_{n}$. Define $\phi: X \rightarrow Y$ by

$$
\begin{aligned}
\phi\left(\left(\frac{1}{n}, \frac{\sin \theta}{n}, \cos \theta\right)\right) & =\left(\frac{1}{n}, \frac{\sin 2 \theta}{n}, \cos 2 \theta\right) \text { and } \\
\phi((0,0, \cos \theta)) & =(0,0, \cos 2 \theta) .
\end{aligned}
$$

One checks readily that $\phi$ is well defined, continuous, 2-to-one and open. To see that $\phi$ is equivariant note that

$$
\begin{aligned}
\phi f_{n} & \left(\left(\frac{1}{n}, \frac{\sin \theta}{n}, \cos \theta\right)\right)=\phi\left(\left(\frac{1}{n}, \frac{-\sin (\theta+\pi / 2)}{n}, \cos (\theta+\pi / 2)\right)\right. \\
& =\left(\frac{1}{n}, \frac{\sin 2 \theta}{n},-\cos 2 \theta\right)=g_{n}\left(\left(\frac{1}{n}, \frac{\sin 2 \theta}{n}, \cos 2 \theta\right)\right. \\
& =g_{n} \phi\left(\left(\frac{1}{n}, \frac{\sin \theta}{n}, \cos \theta\right)\right) .
\end{aligned}
$$

Define $\psi: Y \rightarrow Z$ be projection on the first coordinate. Note that each $g_{n}$ is an isometry, so that $Y$ is almost periodic and hence $\psi$ is an almost periodic extension. Remark that $Y$ is not connected so 2.2 itself is not in danger, but $\psi$ is fiberwise connected locally connected.

We show that $\psi \circ \phi: X \rightarrow Z$ is not almost periodic by showing that $(0,0, \cos (\pi / 4))$ and $(0,0, \cos (3 \pi / 4))$ are regionally proximal with respect to $\psi \circ \phi$. 
Obviously,

$$
\begin{aligned}
\lim _{n \rightarrow \infty}\left(\frac{1}{n}, \frac{\sin ( \pm \pi / 4)}{n}, \cos \pm \frac{\pi}{4}\right) & =\left(0,0, \cos \frac{\pi}{4}\right), \text { but } \\
\lim _{n \rightarrow \infty} f_{n}\left(\left(\frac{1}{n}, \frac{\sin (\pi / 4)}{n}, \cos \frac{\pi}{4}\right)\right) & =\lim _{n \rightarrow \infty}\left(\frac{1}{n}, \frac{-\sin (3 \pi / 4)}{n}, \cos \frac{3 \pi}{4}\right) \\
& =\left(0,0, \cos \frac{3 \pi}{4}\right) \\
\lim _{n \rightarrow \infty} f_{n}\left(\left(\frac{1}{n}, \frac{\sin (-\pi / 4)}{n}, \cos \frac{-\pi}{4}\right)\right) & =\lim _{n \rightarrow \infty}\left(\frac{1}{n}, \frac{-\sin (\pi / 4)}{n}, \cos \frac{\pi}{4}\right) \\
& =\left(0,0, \cos \frac{\pi}{4}\right) .
\end{aligned}
$$

6. Regional proximal relation for suspensions. We give an example of a connected locally almost periodic extension and a 2-to-one extension of minimal flows such that the composition is not locally almost periodic.

To that end we first discuss suspensions, aimed at extending a $\mathbf{Z}$-flow to an $\mathbf{R}$-flow. Then we give an example of a minimal 2-to-one extension of a locally almost periodic flow that is not locally almost periodic. After suspension these flows are connected and still the resulting flow is not locally almost periodic.

This shows that Theorem 4.5 cannot be strengthened to the case where the locally almost periodic extension has connected fibers.

Let $T$ be a topological group and let $S$ be a closed syndetic subgroup of $T$, say $T=K S$ for $K \subseteq T$ compact. Let $\langle S, Y\rangle$ be a flow for $S$. Then also $\langle S, T \times Y\rangle$ is a flow for $S$. Define an equivalence relation $\sim$ on $T \times Y$ by

$$
(t, y) \sim\left(t^{*}, y^{*}\right) \quad \text { iff } t^{-1} t^{*} \in S \text { and } \quad t^{-1} t^{*} y^{*}=y .
$$

[Note that for every $s \in S, t \in T, y \in Y:(t, y) \sim\left(t s, s^{-1} y\right)$.] The suspension $X$ of $Y$ is defined as

$$
\begin{aligned}
& X=T \times Y / \sim \text { notation } X=T \times{ }_{S} Y ; \sim(t, y):=[t, y] . \\
& t^{\prime} \cdot[t, y]:=\left[t^{\prime}, t, y\right] \text { for every } t^{\prime} \in T,[t, y] \in X .
\end{aligned}
$$

Then $\langle T, X\rangle$ is a flow for $T([\mathbf{H} \mathbf{6 0}] \S 5)$.

6.1. REMARK. If $\langle S, Y\rangle$ is minimal then $\left\langle T, T \times{ }_{S} Y\right\rangle$ is minimal.

Proof. Let $[t, y],\left[t^{*}, y^{*}\right] \in T \times{ }_{S} Y$. Let $s_{\lambda} \in S$ be such that $s_{\lambda} y \rightarrow$ $y^{*}$. Then $t^{*} s_{\lambda} t^{-1}[t, y] \rightarrow\left[t^{\prime}, y^{\prime}\right]$, for $t^{*} s_{\lambda} t^{-1}[t, y]=\left[t^{*} s_{\lambda}, y\right]=\left[t^{*}, s_{\lambda} y\right]$. 
The proof of the next lemma is left as an exercise to the reader.

6.2. LEMMA. Let $S$ be a syndetic subgroup of $T$. Let $\langle S, X\rangle$ and $\langle T, X\rangle$ be flows such that the action of $S$ is just a restriction of the action of $T$. Then $Q_{\langle S, X\rangle}=Q_{\langle T, X\rangle}$.

In the sequel we assume $S \subseteq Z(T)$, i.e. $s t=t s$ for every $t \in T$, $s \in S$.

Note that in that case $s[t, y]=[s t, y]=[t s, y]=[t, s y]$.

6.3. Theorem. Let $x_{1}, x_{2} \in X$, say $s_{i}=\left[t_{i}, y_{i}\right]$. Then

$$
\left(x_{1}, x_{2}\right) \in Q_{\langle T, X\rangle} \text { iff } t_{1}^{-1} t_{2}=s \in S \quad \text { and } \quad\left(y_{1}, s y_{2}\right) \in Q_{\langle S, Y\rangle} .
$$

Proof. The if-part is trivial.

Suppose $\left(x_{1}, x_{2}\right) \in Q_{\langle T, X\rangle}$, then, by Lemma 6.2, $\left(x_{1}, x_{2}\right) \in Q_{\langle s, X\rangle}$.

So there are $s_{\lambda} \in S$ and $\left[t_{\lambda}^{i}, y_{\lambda}^{i}\right] \in X, i=1,2$, such that for a certain $[r, \bar{y}] \in X$ :

$$
\left[t_{\lambda}^{i}, y_{\lambda}^{i}\right] \rightarrow\left[t_{i}, y_{i}\right] \text { and } s_{\lambda}\left[t_{\lambda}^{i}, y_{\lambda}^{i}\right] \rightarrow[r, \bar{y}]
$$

As $T=K S$, we can write $t_{\lambda}^{i}=k_{\lambda}^{i} u_{\lambda}^{i}, t_{i}=k_{i} s_{i}$ for $k_{\lambda}^{i}, k_{i} \in K: u_{\lambda}^{i}, s_{i} \in S$. Note that

$$
\left[t_{i}, y_{i}\right]=\left[k_{i}, s_{i} y_{i}\right] ;\left[t_{\lambda}^{i}, y_{\lambda}^{i}\right]=\left[k_{\lambda}^{i}, u_{\lambda}^{i} y_{\lambda}^{i}\right] \rightarrow\left[k_{i}, s_{i} y_{i}\right]
$$

and

$$
s_{\lambda}\left[t_{\lambda}^{i}, y_{\lambda}^{i}\right]=\left[t_{\lambda}^{i}, s_{\lambda} y_{\lambda}^{i}\right]=\left[k_{\lambda}^{i}, u_{\lambda}^{i} s_{\lambda} y_{\lambda}^{i}\right] \rightarrow[r, \bar{y}] .
$$

Since $K$ is compact we may assume, after passing to a suitable subnet,

$$
k_{\lambda}^{i} \rightarrow \bar{k}_{i} \in K ; u_{\lambda}^{i} y_{\lambda}^{i} \rightarrow \bar{y}_{i} \in Y \quad \text { and } \quad u_{\lambda}^{i} s_{\lambda \lambda}^{i} \rightarrow \bar{z}_{i} \in Y .
$$

Then in $T \times Y$ we have

$$
\left(k_{\lambda}^{i}, u_{\lambda}^{i} y_{\lambda}^{i}\right) \rightarrow\left(\bar{k}_{i}, \bar{y}_{i}\right) \text { and }\left(k_{\lambda}^{i}, u_{\lambda}^{i} s_{\lambda} y_{\lambda}^{i}\right) \rightarrow\left(\bar{k}_{i}, \bar{z}_{i}\right) .
$$

Hence, after applying “ ":

$$
\left[t_{i}, y_{i}\right]=\left[k_{i}, s_{i} y_{i}\right]=\left[\bar{k}_{i}, \bar{y}_{i}\right] \text { and }[r, \bar{y}]=\left[\bar{k}_{1}, \bar{z}_{1}\right]=\left[\bar{k}_{2}, \bar{z}_{2}\right] \text {. }
$$

In particular this means that $\bar{k}_{2}=\bar{k}_{1} \bar{s}$ and $\bar{s}_{2}=\bar{z}_{1}$. In $T \times Y$ we have

$$
\begin{aligned}
s_{\lambda}\left(u_{\lambda}^{1} y_{\lambda}^{1}, u_{\lambda}^{2} \bar{s} y_{\lambda}^{2}\right) & =\left(u_{\lambda}^{1} s_{\lambda} y_{\lambda}^{1}, \bar{s} u_{\lambda}^{2} s_{\lambda} y_{\lambda}^{2}\right) \rightarrow\left(\bar{z}_{1}, \bar{s}_{2}\right)=\left(\bar{z}_{1}, \bar{z}_{1}\right), \\
\left(u_{\lambda}^{1} y_{\lambda}, u_{\lambda}^{2} \bar{s} y_{\lambda}^{2}\right) & =\left(u_{\lambda}^{1} y_{\lambda}^{1}, \bar{s} u_{\lambda}^{2} y_{\lambda}^{2}\right) \rightarrow\left(\bar{y}_{1}, \overline{s y}\right) ;
\end{aligned}
$$

which means that $\left(\bar{y}_{1}, \overline{s y}_{2}\right) \in Q_{\langle S, Y\rangle}$. Since $\left[t_{1}, y_{1}\right]=\left[\bar{k}_{1}, \bar{y}_{1}\right]$ and $\left[t_{2}, y_{2}\right]=$ $\left[\bar{k}_{2}, \bar{y}_{2}\right]=\left[\bar{k}_{1} \bar{s}, \bar{y}_{2}\right]=\left[\bar{k}_{1}, \overline{s y}_{2}\right]$, there are $r_{i} \in S$ such that

$$
\bar{k}_{1}=t_{1} r_{1} \quad \text { and } \quad r_{1} \bar{y}_{1}=y_{1}, \quad \bar{k}_{1}=t_{2} r_{2} \quad \text { and } \quad r_{2} \overline{s y}_{2}=y_{2} \text {. }
$$


Hence $t_{2}=\bar{k}_{2} r_{2}^{-1}=t_{1} r_{1} r_{2}^{-1}$, where $r_{1} r_{2}^{-1} \in S$; so $t_{1}^{-1} t_{2}=r_{1} r_{2}^{-1} \in S$. We are left to show that $\left(y_{1}, r_{1} r_{2}^{-1} y_{2}\right) \in Q_{\langle S, Y\rangle}$, as follows. Since $r_{2} \overline{s y}_{2}=y_{2}$, we have $r_{1} r_{2}^{-1} y_{2}=r_{1} r_{2}^{-1} r_{2} \overline{s y}_{2}=r_{1} \overline{s y}_{2}$. So, by the facts

$$
y_{1}=r_{1} \bar{y}_{1} \quad \text { and } \quad\left(\bar{y}_{1}, \overline{s y}_{2}\right) \in Q_{\langle s, Y\rangle}
$$

it follows that

$$
\left(y_{1}, r, r_{2}^{-1} y_{2}\right)=r_{1}\left(\bar{y}_{1}, \overline{s y}_{2}\right) \in Q_{\langle S, Y\rangle} .
$$

In a similar way, but easier, one shows:

6.4. Theorem. Let $x_{1}, x_{2} \in S X$, say $x_{i}=\left[t_{i}, y_{i}\right]$. Then

$$
\left(x_{1}, x_{2}\right) \in P_{\langle T, X\rangle} \quad \text { iff } t_{1}^{-1} t_{2} \in S \quad \text { and } \quad\left(y_{1}, s y_{2}\right) \in P_{\langle S, Y\rangle} .
$$

Let $\phi:\langle S, Y\rangle \rightarrow\langle S, Z\rangle$ be a homomorphism of $S$-flows. Then $\phi$ induces a homomorphism $\hat{\phi}:\left\langle T, T \times{ }_{S} Y\right\rangle \rightarrow\left\langle T, T \times{ }_{S} Z\right\rangle$ defined by

$$
\hat{\phi}[t, y]:=[t, \phi(y)] \text {. }
$$

One checks readily that $\hat{\phi}$ is a well defined continuous and equivariant map.

It is left to the reader to give proofs for the relativized versions of Theorems 6.3 and 6.4. The next corollary is then easily derived:

6.5. Corollary. Let $\phi:\langle S, Y\rangle \rightarrow\langle S, Z\rangle$ be a homomorphism of $S$-flows, and let $\hat{\phi}:\left\langle T, T \times{ }_{S} Y\right\rangle \rightarrow\left\langle T, T \times{ }_{S} Z\right\rangle$ be the induced extension.

(a) If $\phi$ is almost periodic, distal or proximal, so is $\hat{\phi}$. In particular, if $\langle S, Y\rangle$ is almost periodic, then $\left\langle T, T \times{ }_{S} Y\right\rangle$ is almost periodic too.

(b) If $Y$ is minimal, then $\hat{\phi}$ is highly proximal if $\phi$ is.

(c) If $\phi$ is $N$-to-one, $\hat{\phi}$ is $N$-to-one.

After this discussion about suspensions we give an example of a minimal 2-to-one extension of a locally almost periodic flow that is not locally almost periodic:

Let $\langle Y, \phi\rangle$ be the Ellis minimal set ([E 69] 5.29.). As $Y$ is a highly proximal (2-to-one but not open) extension of the circle with irrational rotation, $\langle Y, \phi\rangle$ is locally almost periodic. Let $X=Y \times\{0,1\}$ be the topological sum of two copies of $Y$. 
Define a homeomorphism $\theta$ on $X$ as follows:

Fix a basic clopen subset $W$ of $Y$ such that $\phi[W] \cap W=\varnothing$. Define

$$
\begin{array}{lll}
\theta(y, i)=(\phi(y), 1-i), & i=0,1, & \text { if } y \in W, \\
\theta(y, i)=(\phi(y), i), & i=0,1, & \text { if } y \in W .
\end{array}
$$

then $\xi:\langle X, \theta\rangle \rightarrow\langle Y, \phi\rangle$ is open and 2-to-one, where $\xi$ is defined by $\xi(y, i)=y$.

In order to see that $X$ is minimal, recall that $Y$ consists of two circles, say $S^{+}$and $S^{-}$, and that the basic clopen subset $W$ of $Y$ has the form $\left(a^{+} ; b^{+}\right) \cup\left[a^{-} ; b^{-}\right)$, where $\left(a^{+} ; b^{+}\right)$and $\left[a^{-}, b^{-}\right)$denote intervals in $S^{+}$, $S^{-}$.

Note that $\theta\left(a^{-}, 1\right)=\left(\phi\left(a^{-}\right), 0\right)$ as $a^{-} \in W$ but $\theta\left(a^{+}, 0\right)=\left(\phi\left(a^{+}\right), 0\right)$. Since $Y$ does not have periodic points $\phi\left(a^{-}\right)$and $\phi\left(a^{+}\right)$are both inside or both outside of $W$ under positive powers of $\phi$. So under positive powers of $\theta$ they are in the same $(0$ or 1$)$ level of $X$, hence $\left(a^{-}, 1\right)$ and $\left(a^{+}, 0\right)$ are proximal under positive powers of $\theta$.

Similarly, $\left(a^{+}, 1\right)$ and $\left(a^{-}, 1\right)$ are proximal under negative powers of $\theta$.

6.6. Remark. With notation as above. $X$ is a minimal $\mathbf{Z}$-flow and $X$ is not locally almost periodic.

Proof. Since $Y$ is minimal and $\xi$ is open and two-to-one, $X$ is the union of at most two minimal subsets. If $X$ is not minimal then $X=X_{1}$ $\cup X_{2}$ where $X_{i}$ is minimal.

Since $\left(a^{-}, 1\right)$ is proximal to $\left(a^{+}, 0\right)$ as well as $\left(a^{+}, 1\right)$, all three points have to be in the same minimal subset, say in $X_{1}$. But both $X_{1}$ and $X_{2}$ project onto $Y$; so if $\left(a^{+}, 1\right) \in X_{1},\left(a^{+}, 0\right)$ has to be in $X_{2}$ which is a contradiction. Hence $X$ is minimal.

In order to show that $X$ is not locally almost periodic, it suffices to show that $P_{X}$ is not an equivalence relation. If it were, then $\left(a^{+}, 1\right)$ and $\left(a^{+}, 0\right)$ were proximal, but as they are identified by $\xi$ they are distal.

By now we are able to give the example showing that mere connectedness is not enough in 4.5.:

Let $\langle\mathbf{R}, \hat{X}\rangle,\langle\mathbf{R}, \hat{Y}\rangle$ and $\langle\mathbf{R}, \hat{S}\rangle$ be the suspensions of $\langle X, \theta\rangle,\langle Y, \phi\rangle$ and $\langle S, \alpha\rangle$, where $\langle Y, \phi\rangle \stackrel{n}{\rightarrow}\langle S, \alpha\rangle$ is the highly proximal map of $\langle Y, \phi\rangle$ onto the irrational rotation $\langle S, \alpha\rangle$.

By $6.5, \hat{S}$ is equicontinuous, $\hat{\eta}$ is highly proximal, so $\hat{Y}$ is locally almost periodic and $\hat{\xi}$ is 2-to-one.

Moreover, as $\mathbf{R}$ is connected and $\hat{X}, \hat{Y}$ and $\hat{S}$ are minimal, they are connected. However, as $P_{X}$ is not an equivalence relation, $P_{\hat{X}}$ is not an equivalence relation either. So $\hat{X}$ is not locally almost periodic. 


\section{REFERENCES}

[AG 77] J. Auslander and S. Glasner, Distal and highly proximal extensions of minimal flows, Indiana Math. J., 25 (1977), 731-749.

[AW 81] J. Auslander and J. C. S. P. van der Woude, Maximally highly proximal generators of minimal flows, Ergodic Theory Dynamical Systems, 1 (1981), 389-412.

[B 75/79] I. U. Bronstein, Extensions of minimal transformation groups, Sijthoff \& Noordhoff, Alphen aan den Rijn, 1979 (Russian edition 1975).

[E 69] R. Ellis, Lectures on Topological Dynamics, Benjamin, New York, 1969.

[GH 55] W. H. Gottschalk and G. A. Hedlund, Topological dynamics, Amer. Math. Colloq. Publ., vol. 36, Amer. Math. Soc., Providence, R. I., 1955.

[H 60] F. J. Hahn, Some embeddings, recurrence properties, and the Birkhoff-Markov theorem for transformation groups, Duke Math. J., 27 (1960), 513-525.

[MW 72] D. C. McMahon and T. S. Wu, On weak mixing and local almost periodicity, Duke Math. J., 39 (1972), 333-343.

[MW 76] _ On the connectedness of homomorphisms in topological dynamics, Trans. Amer. Math. Soc., 217 (1976), 257-270.

[MW 80] _ Notes on topological dynamics $I V$ : relative equicontinuity and its variations, Bull. Inst. Math. Acad. Sinica, 8 (1980), 277-281.

[MW 81] _ Distal homomorphisms of nonmetric minimal flows, Proc. Amer. Math. Soc., 82 (1981), 283-294.

[R ?] M. Rees, On the structure of minimal distal transformation groups with manifolds as phase spaces, $\mathrm{Ph}$. D. thesis, Univ. of Warwich, Coventry, England.

[SS 74] R. J. Sacker and G. R. Sell, Finite extensions of minimal transformation groups, Trans. Amer. Math. Soc., 190 (1974), 325-334.

[Sh 76] P. S. Shoenfeld, Highly proximal and generalized almost finite extensions of minimal sets, Pacific J. Math., 66 (1976), 265-280.

[V 77] W. A. Veech, Topological dynamics, Bull. Amer. Math. Soc., 83 (1977), 775-830.

[Wo 82] J. C. S. P. van der Woude, Topological dynamis, Dissertation, Vrije Universiteit, Amsterdam, 1982. CWI Tract Volume 22 1986, Math Centre, Amsterdam.

[W 75] T.-S. Wu, Notes on topological dynamics. II: distal extension with discrete fibers and prime flows, Bull. Inst. Math. Acad. Sinica, 3 (1975), 49-60.

Received March 12, 1985 and in revised form July 31, 1986.

Arizona STATE University

TEMPE, AZ 85281, U.S.A.

TECHNISCHE HogeschoOL EINDHOVEN

EINDHOVEN, THE NETHERLANDS

AND

Case Western Reserve University

Cleveland, OH 44106, U.S.A. 


\section{PACIFIC JOURNAL OF MATHEMATICS EDITORS}

\author{
V. S. VARADARAJAN \\ (Managing Editor) \\ University of California \\ Los Angeles, CA 90024 \\ HERBERT Clemens \\ University of Utah \\ Salt Lake City, UT 84112 \\ R. FINN \\ Stanford University \\ Stanford, CA 94305
}

HERMANN FLASCHKA

University of Arizona

Tucson, AZ 85721

RAMESH A. GANGOLLI

University of Washington

Seattle, WA 98195

VAughan F. R. JONES

University of California

Berkeley, CA 94720

ROBION KIRBY

University of California

Berkeley, CA 94720
C. C. MoOrE

University of California

Berkeley, CA 94720

H. SAMELSON

Stanford University

Stanford, CA 94305

HAROLD STARK

University of California, San Diego

La Jolla, CA 92093

\section{ASSOCIATE EDITORS}
R. AREnS
E. F. BECKENBACH
B. H. NEUMANN
F. WOLF
K. YOSHIDA (1906-1982)

\section{SUPPORTING INSTITUTIONS}

UNIVERSITY OF ARIZONA

UNIVERSITY OF BRITISH COLUMBIA

CALIFORNIA INSTITUTE OF TECHNOLOGY

UNIVERSITY OF CALIFORNIA

MONTANA STATE UNIVERSITY

UNIVERSITY OF NEVADA, RENO

NEW MEXICO STATE UNIVERSITY

OREGON STATE UNIVERSITY
UNIVERSITY OF OREGON UNIVERSITY OF SOUTHERN CALIFORNIA STANFORD UNIVERSITY UNIVERSITY OF HAWAII UNIVERSITY OF TOKYO UNIVERSITY OF UTAH WASHINGTON STATE UNIVERSITY UNIVERSITY OF WASHINGTON 


\section{Pacific Journal of Mathematics}

\section{Vol. 128, No. $1 \quad$ March, 1987}

Anthony Peter Bahri and Peter Gilkey, The eta invariant, $\operatorname{Pin}^{c}$ bordism, and equivariant $\operatorname{Spin}^{c}$ bordism for cyclic 2-groups $\ldots \ldots \ldots \ldots \ldots \ldots 1$

Friedrich-Wilhelm Bauer, Extensions of generalized homology theories . . . 25

Marilyn Breen, A characterization theorem for compact unions of two

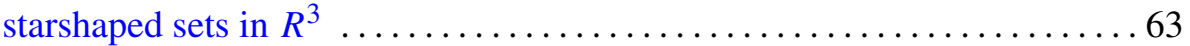

Boniface Ihemotuonye Eke, Special generating sets of purely inseparable extension fields of unbounded exponent $\ldots \ldots \ldots \ldots \ldots \ldots \ldots \ldots \ldots \ldots$

Robert William Gilmer, Jr. and William James Heinzer, Jónsson $\omega_{0}$-generated algebraic field extensions $\ldots \ldots \ldots \ldots \ldots \ldots \ldots \ldots \ldots . . .61$

Guido Lupacciolu, Holomorphic continuation in several complex variables

Douglas C. McMahon, Jaap C. S. P. van der Woude and Ta-Sun Wu,

Connectedness related to almost periodicity of compositions of flow

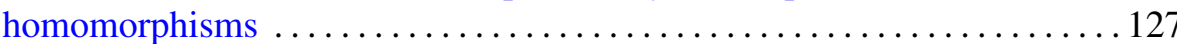

Sophocles Mercourakis, Some characterizations of analytic metric spaces

Peter Frederick Stiller, The Picard numbers of elliptic surfaces with many

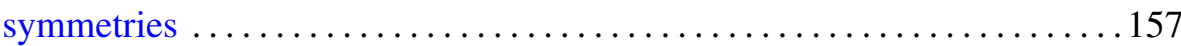

David J. Winter, Reducible complements of Lie algebra radicals ........ 191 Rade Živaljević, On a cohomology theory based on hyperfinite sums of microsimplexes 